\title{
brainR: Interactive 3 and 4D Images of High Resolution Neuroimage Data
}

by John Muschelli, Elizabeth Sweeney, and Ciprian Crainiceanu

Abstract We provide software tools for displaying and publishing interactive 3-dimensional (3D) and 4-dimensional (4D) figures to html webpages, with examples of high-resolution brain imaging. Our framework is based in the R statistical software using the rgl package, a 3D graphics library. We build on this package to allow manipulation of figures including rotation and translation, zooming, coloring of brain substructures, adjusting transparency levels, and addition/or removal of brain structures. The need for better visualization tools of ultra high dimensional data is ever present; we are providing a clean, simple, web-based option. We also provide a package (brainR) for users to readily implement these tools.

\section{Introduction}

We provide a set of software tools that produce interactive 3-dimensional (3D) and 4-dimensional (4D) figures using currently available open-source software. These images can be embedded into webpages (http://bit.1y/1kEJH6q), creating an environment where users can interact with the images without additional software or expertise. We present two examples of applications of these tools using neuroimaging data: 1) a 3D example: displaying hyper-intense white matter regions in a template image; and 2) a 4D example: tracking the lesion formation process over time via 3D segmentation.

Many applications display true 3D data as single 2-dimensional (2D) cross-sections, a series of cross-sections, or a projection of the data onto 2D space. In neuroimaging data, for example, the current standard is to present spatial results as a set of $2 \mathrm{D}$ figures, or slices. Figure 1 displays the magnetic resonance image (MRI) of a normal brain and includes three planar views: axial (vertical plane dividing the body into front and back), sagittal (vertical plane dividing the brain into right and left), and coronal (horizontal plane dividing the brain into top and bottom). Other types of presentations can be employed, such as lightbox views where all slices of the brain are presented in series, single plane views, 3D surface images, or maximum intensity projections onto transparent cross-sectional views (known as "glass brains", Figure 1B). We wish to improve on these data representations by using truly 3D figures.
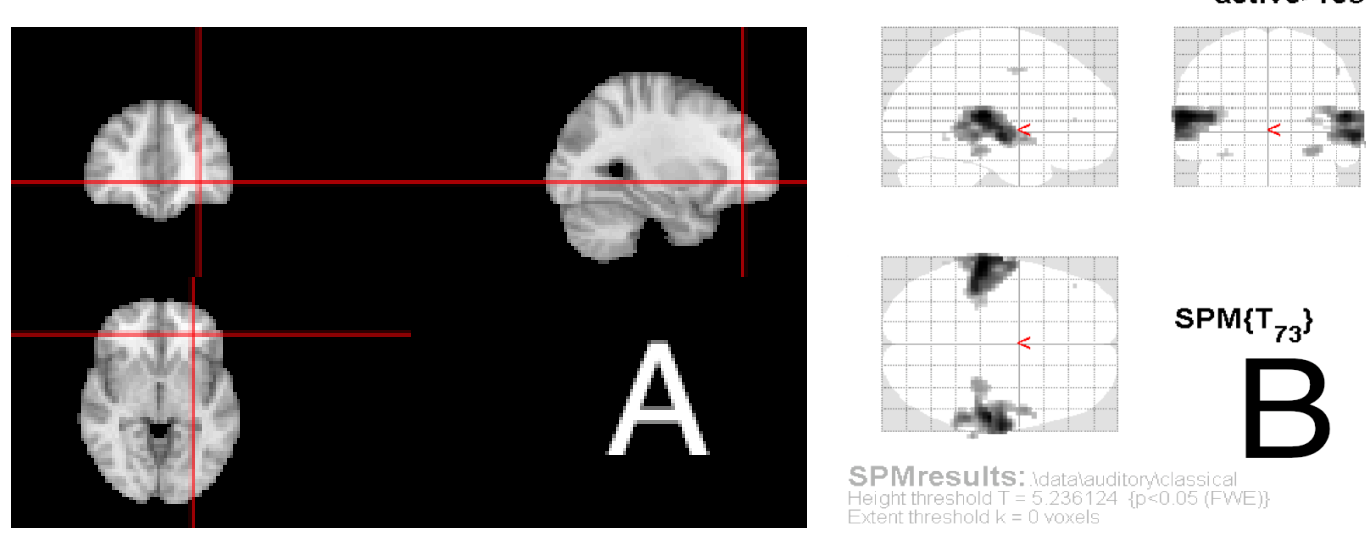

Figure 1: Example of an othrographic representation of the brain (A), where the brain is displayed axially (bottom), coronally (top left), and sagitally (top right). Cross-hairs (red) are shown to orient the user to how certain areas fall in each dimension. Figures using maximum intensity projections show a similar set of 3 transparent views, known as "glass brains", such as (B) from the SPM8 manual, showing how certain information (in this case activation) corresponds spatially (Ashburner et al., 2008).

The goal of these multi-panel 2D figures is to allow the user to: 1) orient themselves spatially; 2) understand the local brain features or pathology in relationship to known and observable biological landmarks; and 3) provide qualitative or quantitative assessments of the image. While these approaches provide important information and are extensively used in practice, they also have innate limitations. For example, the slices presented must be chosen by the presenter/author, which may not 
be representative of all areas of the brain. Most importantly though, the true 3D structure of the data is not exploited and $4 \mathrm{D}$ interactive visualizations are difficult to implement.

With the advancement of $3 \mathrm{D}$ rendering techniques and web-based tools, the ability to provide more intuitive and informative figures is now possible. People are not yet taking full advantage of the ability to present true, interactive 3D figures. This may be because 3D figures are not currently accepted for publication, a fact that is likely to change with the generational shift to digital journal print. Therefore, the purpose of this article is to inform the reader how to create and use interactive 3D figures for exploration, presentation, and published-article figures.

We will be providing 1) the characteristics of a good $4 \mathrm{D}$ interactive figure, 2) a framework for creating these figures in $R, 3$ ) examples of creating figures within this framework using $R$, and 4) description of practical applications for these figures. The examples in the article are brain images, but these concepts and methods can be used for general 3D surfaces.

\section{What makes a good 4D interactive figure?}

In order to determine software necessary for 3D figure generation, we discuss properties of the figures and the process that are required for their generation. We argue that for the interactive capabilities of $3 \mathrm{D}$ figures to have added value over current presentation methods they should have the following characteristics:

- Rotation/Translation: allows the user to see objects from multiple angles and positions.

- Digital zoom: enables multilayer display of information from minute details to coarser anatomy.

- Color: allows for proper contrast between structures, and also the ability to highlight regions of interest.

- Transparency: provides the ability to adaptively explore the changing opacity of cortical surface as well as sub-cortical deep structures.

- Addition/Removal of structures: allows users to contrast one surface with another by adding or removing an individual surface to see how the surfaces overlap and compare spatially.

These capabilities provide users the ability to more accurately view differences between populations, image processing pipelines, and segmentation algorithms. With the addition and removal of surfaces, users can also view changes in brain structures longitudinally in 4D.

We refer to $4 \mathrm{D}$ viewing as observing 3D objects over another domain other than space, such as time. The user interacts over this domain while keeping the brain in the same 3D space. For example, characterizing the size and shape changes of the hippocampus over time within a person. Similarly, activation maps derived from a functional MRI (fMRI) task can be calculated for a diseased group of people and a control group. We can present the area of activation from the control group and switch to the map for a diseased group for comparison. Lastly, we have used these techniques to determine how a hemorrhage in the brain changes over time-pre and post treatment-depicting where the treatment had removed hemorrhage.

Even with these capabilities, without quick and easy usability, sophisticated plotting tools, such as those described here, will not be used in practice. Therefore, the publishing platform needs to render surfaces very fast and be easy to use and manipulate. Publishing and presenting such figures in a user-friendly interface that does not require third-party software is another essential part of visualization.

\section{Software and tools}

Many rendering systems, or analysis tools with rendering capabilities exist, such as FreeSurfer (http: //surfer. nmr. mgh . harvard. edu/), ParaView (Henderson et al., 2004), MIPAV (McAuliffe et al., 2013), 3D Slicer (Pieper et al., 2004), and rg1 (Adler and Murdoch, 2014) in R. We will focus on the R package rgl as it has the desired characteristics outlined above and can easily export images to the web with a small amount of setup and knowledge of the system.

The R programming language is becoming more widely used, with expanding areas of research and capabilities. $\mathbf{r g l}$ is an $\mathrm{R}$ implementation of the graphics programming interface Open Graphics Library (OpenGL; Shreiner et al., 2009), that allows for 3D rendering. These rendered objects can be interactively explored on screen. We prefer using R because it has many packages that read, analyze, and display neuroimaging data (Whitcher et al. 2011; Tabelow and Polzehl 2011; Bordier et al. 2011, see also the CRAN Task View on Medical Image Analysis; Whitcher 2013), is multi-platform, free, and open-source with a large user and developer community. As a statistical programming language, it contains state-of-the-art statistical tools with a growing number of packages for additional analysis, 
and the capability to create highly customizable figures. Our framework uses rgl to generate the figures and export these figures as webpages using WebGL, the web implementation of OpenGL. Using these objects and the X Toolkit (XTK; https://github.com/xtk/X), one can create customizable webpages with versatile user-interaction capabilities. Moreover, $\mathrm{R}$ also has integrated tools such as knitr and Sweave that allow the user to completely reproduce a figure and analysis, and slidify to create HTML5 slide decks with these figures embedded (Xie, 2014; Leisch, 2002; Vaidyanathan, 2012).

Levine et al. (2010) presented a way to embed 3D objects from rgl directly into a Portable Document Format (PDF) document, along with additional applications (Bowman and Shardt, 2009). Though this option is promising, we have found that large images, such as Figure 3 do not render quickly and can lag when interacting with them on a laptop and sometimes crash before rendering even with moderate amounts of memory (Mac OSX 10.9.2, 16Gb RAM, 2.8GHz Intel i7). Furthermore, all capabilities mentioned above were not implemented, such as interactively changing transparency or $4 \mathrm{D}$ visualization, and customized control is not easily implemented in the framework of Levine et al. (2010) without learning additional graphics languages. Some of these are current limitations of the interactive capabilities within PDF, but the integration of JavaScript into PDF documents will likely expand on these capabilities (Story, 2001). We acknowledge others have proposed similar ideas of 3D rendering in R using Virtual Reality Markup Language (VRML; Glaab et al., 2010). We chose to focus on WebGL because browsers require VRML viewer browser plugins and popular browsers (Safari, Google Chrome, Firefox) can render WebGL without any additional plugins.

Although users may prefer some of the aforementioned systems, we wish to inform the community about a simple option to create these figures; we also discuss why they should be more generally used and accepted for publication.

\section{Framework}

Figure 2 illustrates our working framework for figure generation. Briefly, the neuroimaging data is processed and areas of interest are extracted. Examples include: activation maps from fMRI studies during tasks, parcellations of cortical and deep structures, segmentation maps for detection of abnormal tissue, and more general regions of interest (ROIs). These images are passed into the rendering system and surfaces are created from either binary masks or thresholds of the image values. A surface is a collection of vertices that are connected. A collection of multiple surfaces together will be referred to as a scene.

First, the misc3d package creates 3D contours for the surfaces for rendering (Feng and Tierney, 2008). Once the surface is created, $\mathbf{r g l}$ can create and export the scene in two ways: 1 ) output all properties of the scene (colors, shading and lighting, surface orientation) and vertices of the surface directly into one html file or 2) export the vertices of a surface into a 3D-object file and separately write the scene properties into the html file. Option 1 is easier and is our recommendation for exporting 3D images. This process uses the writeWebGL command from the rgl package and creates one html file with an accompanying JavaScript library. An example of an exported figure is shown in Figure 3, which depicts a 3D rendering of a brain template (Grabner et al., 2006) and areas of hyper-intense white matter in WebGL.

One problem with this option is that surfaces in WebGL are limited to 65535 vertices, which may be too few for some large figures that are common in high resolution imaging data. To avoid this, users can downsample their data to reduce the dimensions or use writeWebGL_split: a simple function to split the data into smaller structures so that these larger images can be displayed (courtesy of Duncan Murdoch). This function is located in the brain R package. Although easily executed, this option is limited in 2 ways: 1) the html file contains all vertices of a surface; there can be thousands of vertices-this limits the readability of the html file and 2) it does not easily allow for adding user control of surfaces to create a $4 \mathrm{D}$ figure.

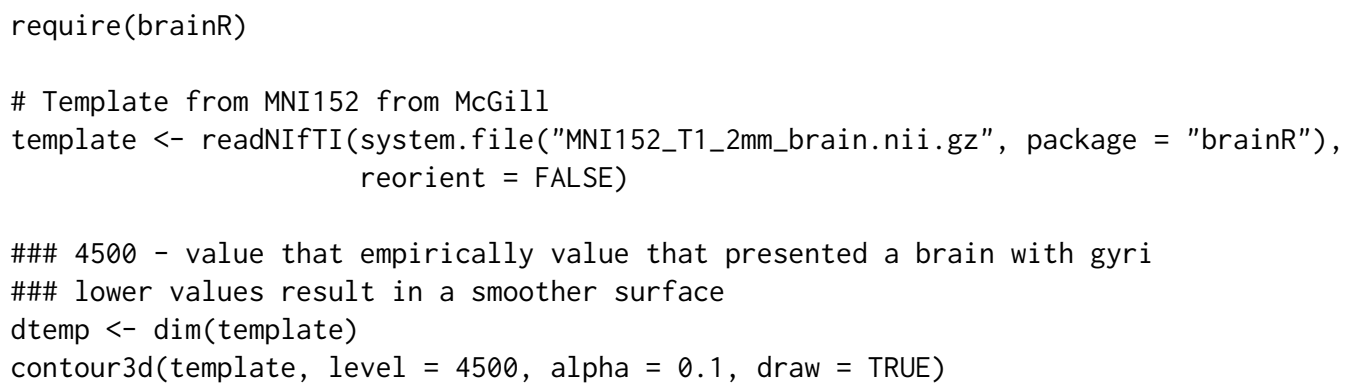




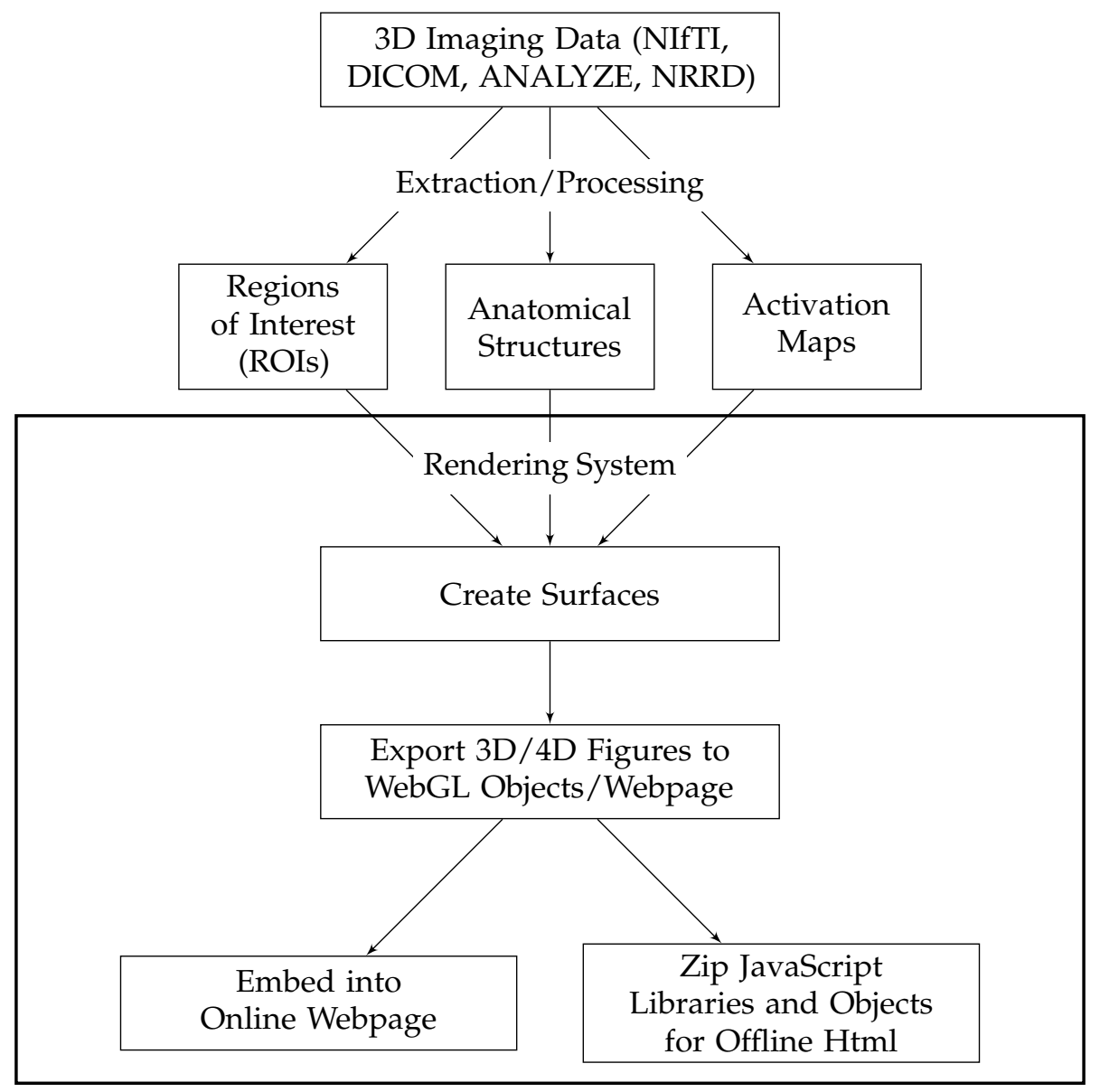

Figure 2: Overall workflow of generating 3D or 4D figures from data. After the raw data has been processed and areas have been extracted, surfaces can be rendered. We are concerned with the steps outlined in the large box: creating surfaces and export to the web. The last branch shows 2 options for export: publishing the figure to the web or enclosing it in a folder with all other libraries needed for rendering. The second option allows users to include these zipped directories as supplementary figures (3D and 4D interactive images are not currently published by journals).

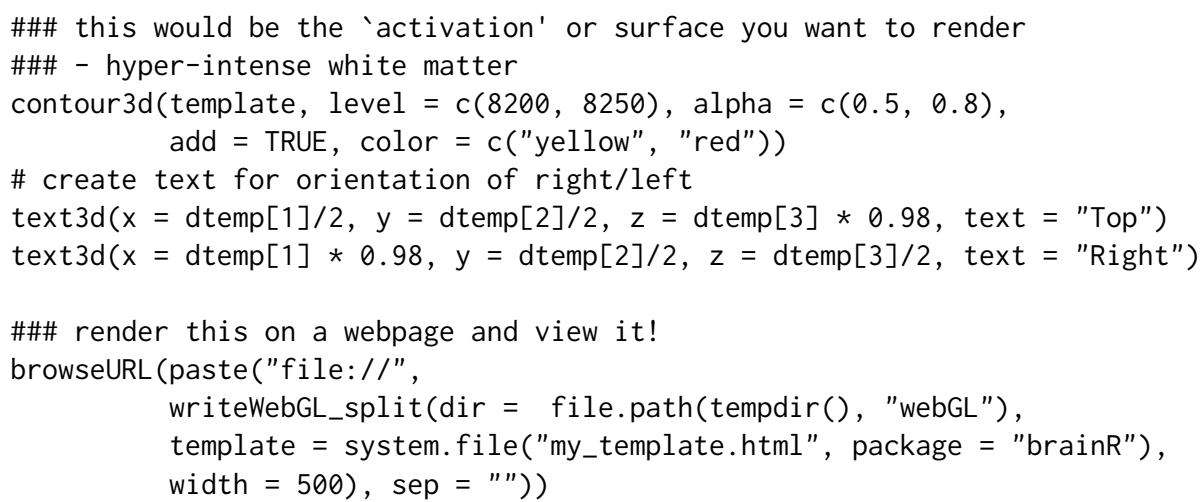

Option 2 allows users to export individual objects/surfaces to either STL (STereoLithography) or Wavefront OBJ files-file formats for 3D surfaces. XTK renders these individual surfaces, and users interact with these objects by turning surfaces on or off, changing opacity, or manipulating any of the surface attributes interactively on the webpage with JavaScript. This process produces interactive $4 \mathrm{D}$ figures, allowing 3D brain structures to be contrasted such as parcellations of the same brain area under different algorithms or single-subject stroke segmentations over time.

We have included functions (write4D and write4D. file) in the brainR package that allow users to export a scene and embed it in html with basic JavaScript controls. We have included a 4D example of segmentation of white matter lesions in a patient with multiple sclerosis (MS) using SubLIME, an automated method for segmenting enlarging and incident MS lesions (Sweeney et al., 2012). Each 


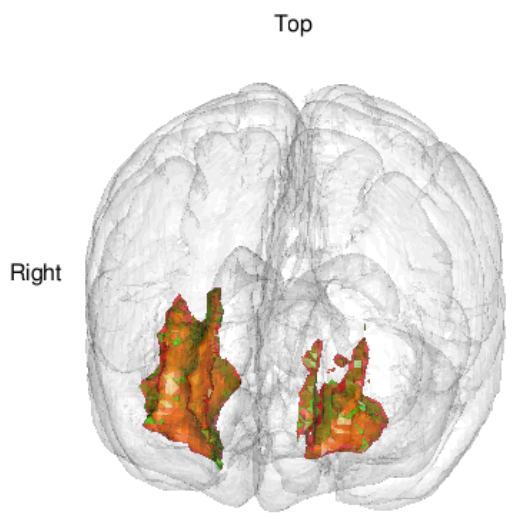

Figure 3: Snapshot of a 3D interactive figure exported from $\mathrm{R}$ using writeWebGL. The figure has many of the qualities of a good figure: transparency, text rendering, colors, and multiple surfaces. The online web version is located http://bit.ly/QrJqs2 (high-resolution version). Viewing the page source code illustrates how all points of the surfaces are outputted directly to html-rendering users unable to easily replicate the figure without source code. Full code is located at http://bit. ly/1gE48M1.

surface represents enlarging lesions compared to the last visit. The rendered html allows control of the opacity of the brain and to compare which areas of the brain have lesion enlargements over time (Figure 4). This $4 \mathrm{D}$ rendering of new and enlarging MS lesions could be used to monitor the clinical progression of the disease.

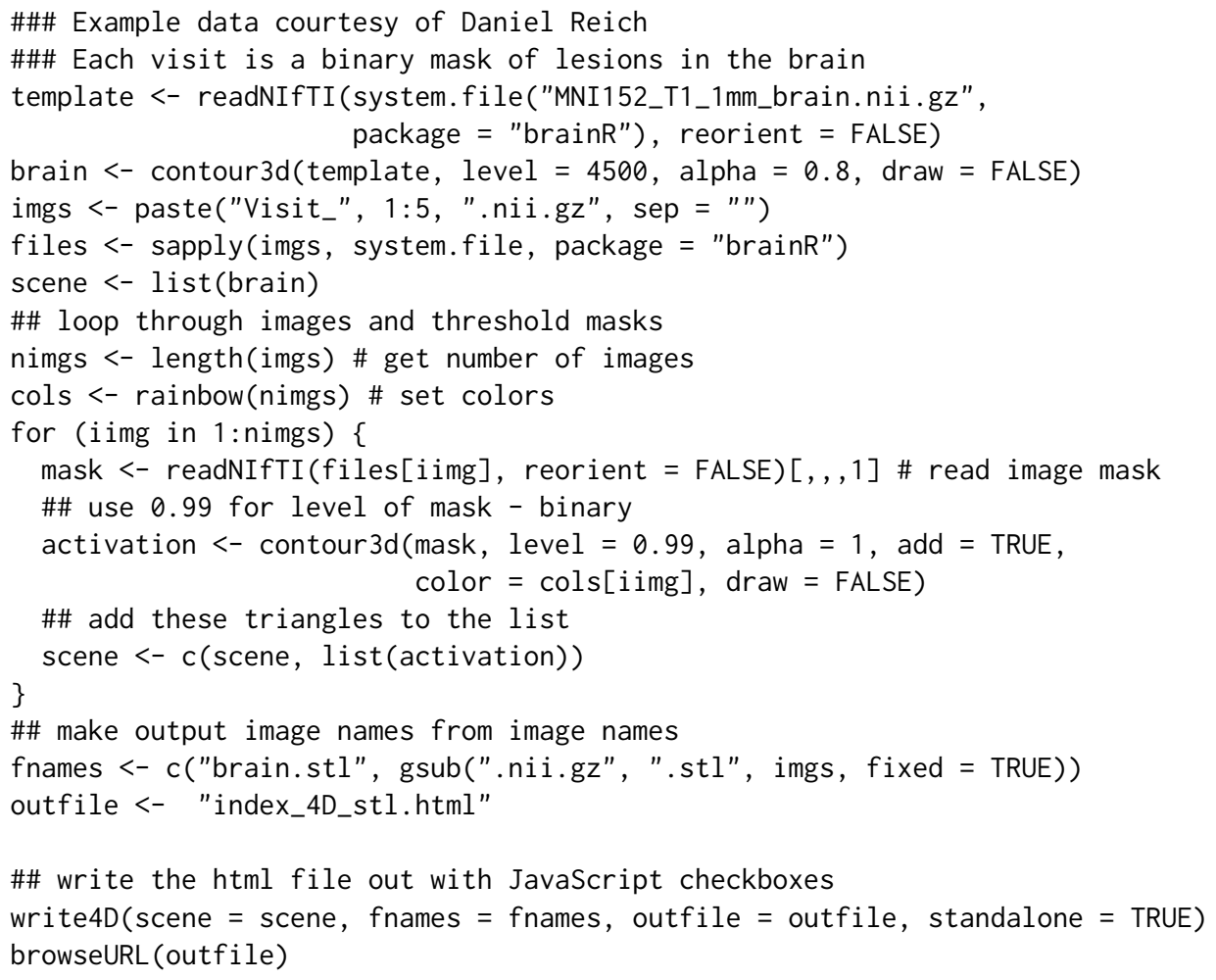

\section{Discussion}

\section{Webpages as a medium}

Our main proposal is to create interactive 3D and 4D figures that allow easy manipulation of embedded $3 \mathrm{D}$ objects and additional annotations. We propose export directly to webpages, which makes the figures widely available, reduces dependence on operating systems, and allows a much larger community to be able to interact with the data. 


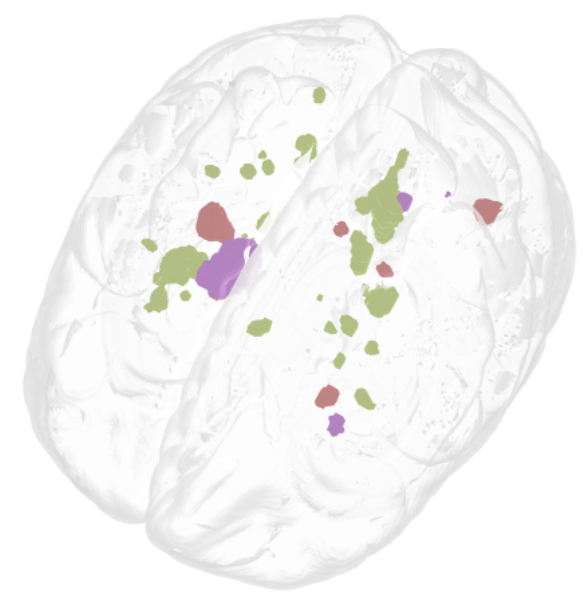

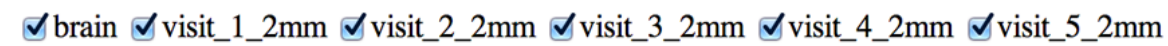

Brain Alpha Blending Slider $\longrightarrow 0.61$

Figure 4: Example of a 4D Figure: a 3D rendered brain (gray) and areas of enlarging white matter lesions in a patient with MS over different followup visits (multiple colors). The checkboxes switch between lesion enlargements at different followup visits and can display multiple visits simultaneously. The brain alpha blending slider (bottom left) allows you to change the opacity of the brain image; this feature can also be added for each surface. We observe that lesions enlarge at many different regions over different visits. The interactive version is located at http://bit. 1y/1 kEJH6q, and code http://bit.1y/1kdnWeg.

Browser interfaces are versatile, flexible, and have large support communities, but rendering may rely on libraries that are not maintained by researchers. Thus, their maintenance is not guaranteed, but the likelihood of maintenance is higher with increased use. The main concern is to produce figures under JavaScript libraries and R packages that are popular and therefore more likely to be updated and improved. Also, including these libraries in the document as supplementary material for standalone figures allows for authors to guarantee which versions of the software readers will be using.

Although highly versatile, web-based tools are not browser independent. Some 3D and 4D interactive figures may be supported on some browsers but not others and may not be usable on the expanding number of devices, such as tablets or smart phones. Our current software works with browsers supporting WebGL and HTML5. Even with these limitations, webpages as figures are extremely powerful and adaptable and present the basic avenue for easy-to-use interactive figures.

\section{Embedding figures into PDF documents}

Previous authors have contributed work that allow users to embed 3D figures directly into PDF documents (Levine et al., 2010; Barnes et al., 2013). While we think this is a useful endeavor for many applications, some of the capabilities described above are lacking. Another drawback is that predominantly all rendering must be done within the Adobe Acrobat Reader (Adobe Systems, 2014), as it is the primary PDF reader with 3D capabilities. Also, we have found that web-based rendering performs much faster than those within PDF for the dimensionality of points we are plotting. Overall, we think this system may fully integrate the needs of the end user into one document, but currently is not optimal for 3D and 4D neuroimaging figures.

\section{Practical applications of 3/4D figures}

Above, we described how this framework has been used to view a longitudinal image segmentation in a holistic way for scans of patients with MS. Other potential applications for this framework are exploratory data analysis and communication of analysis results. The results from analysis such as segmentation, thresholding, or any other surface-generating mechanism can be easily viewed in 3D. Parameters from an analysis can be interactively changed and optimized in this framework. Also, as these figures are in webpages, embedding them into online presentations or journal articles is straightforward and simple. Therefore, journals will not be required to change the way they publish articles while still allowing audiences to interact with figures and get a more comprehensive view of results. 


\section{Conclusion}

Interactive 3D figures can be created quickly. They are reproducible and can be exported easily to standalone webpages. This framework allows for these figures to supplement $2 \mathrm{D}$ figures to present results from analysis and image processing in neuroimaging research. These figures allow users to view the data in the natural setting: 3D space. Users can also create 4D images that can spatially display longitudinal data, group comparisons of areas of activation, differences between parcellation schemes, different independent component analysis networks, and other results from neuroimaging analysis. This provides a powerful alternative to the current state-of-the-art, which relies predominantly on projections, multiple figures, or other reductions of data that make comparisons more difficult than necessary. To our knowledge we are the first to present a framework for depicting neuroimaging results in a condensed, interactive figure with the aforementioned capabilities.

\section{Sources of funding}

The project described was supported by the National Institutes of Health (NIH) grant RO1EB012547 from the National Institute of Biomedical Imaging And Bioengineering, training grant T32AG000247 from the National Institute on Aging, NIH grants RO1NS060910 and RO1NS085211 from the National Institute of Neurological Disorders and Stroke (NINDS), and by NIH grant RO1MH095836 from the National Institute of Mental Health.

\section{Acknowledgements}

The authors thank Brian Caffo, Martin Lindquist, and Duncan Murdoch for comments/help and Daniel Reich for providing the MS lesion data.

\section{Supplemental material}

\section{Enabling WebGL in Safari}

By default, WebGL is not enabled in Safari. This is a description of how to enable WebGL in Safari in order to view the webpages created in this paper.

In Safari, open the Safari menu and select Preferences. Then, click the Advanced tab in the Preferences window. Then, at the bottom of the window, check the Show Develop menu in the menu bar checkbox. Then, open the Develop menu in the menu bar and select Enable WebGL.

\section{Bibliography}

D. Adler and D. Murdoch. rgl: 3D Visualization Device System (OpenGL), 2014. URL http://CRAN. Rproject. org/package=rgl. R package version 0.93.996. [p42]

Adobe Systems. Adobe acrobat reader, 2014. URL www. adobe. com/acrobat. [p46]

J. Ashburner, G. Barnes, C. Chen, J. Daunizeau, G. Flandin, K. Friston, D. Gitelman, S. Kiebel, J. Kilner, V. Litvak, et al. SPM8 Manual. Functional Imaging Laboratory, Institute of Neurology, 2008. [p41]

D. G. Barnes, M. Vidiassov, B. Ruthensteiner, C. J. Fluke, M. R. Quayle, and C. R. McHenry. Embedding and publishing interactive, 3-dimensional, scientific figures in portable document format (PDF) files. PloS ONE, 8(9):e69446, 2013. [p46]

C. Bordier, M. Dojat, and P. L. de Micheaux. Temporal and spatial independent component analysis for fMRI data sets embedded in the AnalyzeFMRI R package. Journal of Statistical Software, 44(9): 1-24, 2011. URL http://www. jstatsoft.org/v44/i09/. [p42]

J. C. Bowman and O. Shardt. Asymptote: Lifting TeX to three dimensions. TUGboat: The Communications of the TEX Users Group, 30(1):58-63, 2009. [p43]

D. Feng and L. Tierney. Computing and displaying isosurfaces in R. Journal of Statistical Software, 28(1), 2008. URL http://www. jstatsoft.org/v28/i01/. [p43]

E. Glaab, J. Garibaldi, and N. Krasnogor. vrmlgen: An R package for 3d data visualization on the web. Journal of Statistical Software, 36(8):1-18, 2010. URL http://www. jstatsoft. org/v36/i08/. [p43] 
G. Grabner, A. L. Janke, M. M. Budge, D. Smith, J. Pruessner, and D. L. Collins. Symmetric atlasing and model based segmentation: An application to the hippocampus in older adults. In Medical Image Computing and Computer-Assisted Intervention-MICCAI 2006, pages 58-66. Springer, 2006. [p43]

A. Henderson, J. Ahrens, and C. Law. The ParaView Guide. Kitware, Clifton Park, NY, 2004. [p42]

F. Leisch. Sweave: Dynamic generation of statistical reports using literate data analysis. In COMPSTAT 2002-Proceedings in Computational Statistics, pages 575-580, Heidelberg, 2002. Physica-Verlag. [p43]

R. A. Levine, L. Tierney, H. Wickham, E. Sampson, D. Cook, and D. A. van Dyk. Editorial: Publishing animations, 3d visualizations, and movies in JCGS. Journal of Computational and Graphical Statistics, 19(1):1-2, 2010. [p43, 46]

M. McAuliffe, P. Bazin, A. Bokinsky, R. Cheng, B. Gandler, E. McCreedy, J. Senseney, and B. Tyrie. MIPAV: Medical Image Processing, Analysis, and Visualization (Version 7.0.1) [Software], 2013. Available from http://mipav.cit.nih.gov/. [p42]

S. Pieper, M. Halle, and R. Kikinis. 3D slicer. In IEEE International Symposium on Biomedical Imaging: Nano to Macro, 2004, volume 1, pages 632-635, Apr. 2004. [p42]

D. Shreiner et al. OpenGL Programming Guide: The Official Guide to Learning OpenGL, Versions 3.0 and 3.1. Addison-Wesley Professional, 2009. [p42]

D. Story. Techniques of introducing document-level JavaScript into a PDF file from a LaTeX source. TUGboat: The Communications of the TEX Users Group, 22(3):161-167, 2001. [p43]

E. Sweeney, R. Shinohara, C. Shea, D. Reich, and C. Crainiceanu. Automatic lesion incidence estimation and detection in multiple sclerosis using multisequence longitudinal MRI. American Journal of Neuroradiology, 34(1):68-73, 2012. [p44]

K. Tabelow and J. Polzehl. Statistical parametric maps for functional MRI experiments in R: The package fmri. Journal of Statistical Software, 44(11):1-21, 2011. URL http://www. jstatsoft. org/ $\mathrm{v} 44 / \mathrm{i} 11 / .[\mathrm{p} 42]$

R. Vaidyanathan. slidify: Generate reproducible html5 slides from $R$ markdown, 2012. URL http:// ramnathv.github.com/slidify/. R package version 0.3.3. [p43]

B. Whitcher. CRAN task view: Medical image analysis, 2013. URL http://CRAN.R-project.org/ view=Medical Imaging. Version 2013-08-12. [p42]

B. Whitcher, V. J. Schmid, and A. Thornton. Working with the DICOM and NIfTI data standards in R. Journal of Statistical Software, 44(6):1-28, 2011. URL http://www. jstatsoft. org/v44/i06/. [p42]

Y. Xie. knitr: A general-purpose package for dynamic report generation in R, 2014. URL http: //yi hui . name/ $\mathrm{knitr/.} \mathrm{R} \mathrm{package} \mathrm{version} \mathrm{1.6.} \mathrm{[p43]}$

John Muschelli

PhD Student

Johns Hopkins Bloomberg School of Public Health,

Baltimore, MD 21231

USA jmuschel@jhsph.edu

Elizabeth Sweeney

PhD Student

Johns Hopkins Bloomberg School of Public Health

Baltimore, MD 21231

USA

Special Volunteer Translational Neuroradiology Unit, Neuroimmunology Branch, National Institute of Neurological Disease and Stroke, National Institute of Health

Bethesda, MD 20892

USA emsweene@jhsph.edu

Ciprian Crainiceanu

Professor

Johns Hopkins Bloomberg School of Public Health,

Baltimore, MD 21231

USA ccrainic@jhsph.edu 